\title{
QUISTE EPIDERMOIDE TESTICULAR BILATERAL EN UN PACIENTE PEDIÁTRICO CON SÍNDROME DE KLINEFELTER
}

\author{
J.M. ALAPONT ALACREU*, C. DOMÍNGUEZ HINAREJOS*, A. SERRANO DURBÁ*, \\ F. ESTORNELL*, M. MARTÍNEZ VERDUCH*, F. VERA SEMPERE**, F. MORENO***, \\ F. GARCÍA IBARRA*
}

*Servicio de Urología Infantil. **Anatomía Patológica. ***Endocrinología Infantil. Hospital Universitario La Fe. Valencia.

Actas Urol Esp. 27 (9): 742-744, 2003

\section{RESUMEN}

QUISTE EPIDERMOIDE TESTICULAR BILATERAL EN UN PACIENTE PEDIÁTRICO CON SÍNDROME DE KLINEFELTER

Los quistes epidérmicos testiculares son lesiones muy infrecuentes en la edad infantil (3\% de todas las tumoraciones testiculares). Sólo se ha descrito su aparición bilateral en la edad pediátrica en 2 observaciones y ninguna de ellas asociada a sindrome de Klinefelter.

Presentamos, en este sentido, el primer caso en nuestro conocimiento de quiste epidérmico bilateral testicular asociado a síndrome de Klinefelter en un niño, destacando el manejo y la conducta terapéutica realizada analizando asimismo las distintas pautas de tratamiento propuestas.

PALABRAS CLAVE: Quiste epidermoide. Síndrome de Klinefelter.

\section{ABSTRACT}

BILATERAL EPIDERMOID TESTICULAR CYST IN AN INFANT WITH KLINEFELTER'S SYNDROME

Epidermoid cysts of the testis are rare in children (3\% of all the testicular tumors). Bilateral appearance has only been described in the pediatric age in 2 cases and none associated to Klinefelter's syndrome.

We present, for our knowledge, the first case of bilateral epidermoid testicular cyst associated to klinefelter's syndrome in a boy, highlighting its management and therapeutic approach. We analyze the different kinds of treatment.

KEY WORDS: Epidermoid cyst. Klinefelter's syndrome.

$\mathrm{L}$ os tumores testiculares son infrecuentes en la ledad pediátrica, siendo la incidencia de 0,5 $2 / 100.000$ niños. De éstos tan sólo el $3 \%^{1}$ son quistes epidermoides por lo que podemos decir que se trata de una patología muy infrecuente en el niño. Se han descrito 5 casos bilaterales y sólo 2 en pacientes menores de 15 años (ambos tratados con orquiectomía parcial). Presentamos un caso de quiste epidérmico bilateral testicular en un niño con síndrome de Klinefelter.

\section{CASO CLÍNICO}

Varón con antecedentes de herniorrafia inguinal bilateral a los 3 meses de edad durante la cual se detectaron unos testes aumentados de tamaño, por lo que se decidió realizar biopsia con resultado de quistes epidérmicos. A los 2 años se produce un aumento considerable del teste izquierdo, motivo por el que es remitido a urología. A la exploración presenta una masa indolora en hemiescroto izquierdo que parece depender 
del testículo. La ecografía (Fig. 1) objetiva una desestructuración testicular, con aumento del tamaño de ambos testes. La alfafetoproteína, testosterona y b-coriogonadotropina humana en suero fueron normales. Ante la sospecha de indiferenciación gonadal solicitamos cariotipo que reveló un síndrome de Klinefelter (47XXY). Dado el resultado de la biopsia previa optamos por actitud expectante. Ante un nuevo aumento del teste izquierdo 2 años después y realizar ecografía de control (Fig. 2) se decidió practicar nueva exploración quirúrgica. Tras acceso inguinal y ligadura del cordón espermático realizamos biopsia testicular extemporánea por congelación, que se informó como lesión testicular sospechosa de quiste epidérmico por lo que decidimos seguir conducta conservadora. El estudio histológico tras inclusión en parafina del material biópsico confirmó la naturaleza epidermoide (Fig. 3). Tras

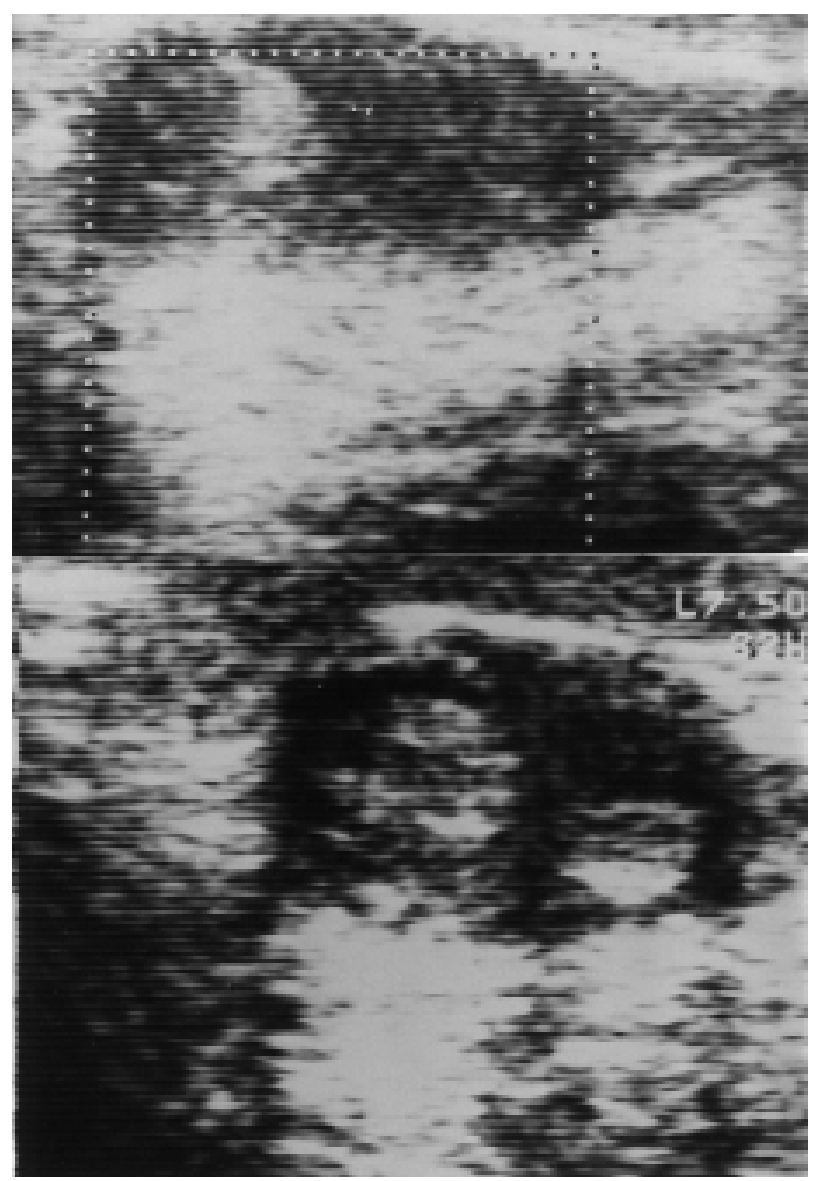

FIGURA 1. Ecografia escrotal: aumento de tamaño testicular con áreas redondeadas hiperecogénicas intraparenquimatosas.
7 años de seguimiento y a la edad de 11 años no se ha producido ningún cambio testicular y el paciente permanece asintomático.

\section{DISCUSIÓN}

Los quistes epidermoides testiculares son tumoraciones benignas sin potencial metastásico que se presentan como masas intratesticulares asintomáticas, induradas y lisas de entre 0,5 y 4,5 $\mathrm{cm}$ de tamaño. La etiología es controvertida aunque la hipótesis más aceptada es que representa un teratoma unilateral o monodérmico benigno ${ }^{2}$.

Los pacientes con síndrome de Klinefelter presentan una mayor predisposición a desarrollar especialmente tumores de células germinales, en particular de origen extragonadal (coriocarcinoma), sin embargo tan sólo se han descrito 3 casos asociados a este síndrome y ninguno bilateral ${ }^{3,4}$, no estableciéndose la relación que pudiera existir. El caso que aportamos se da en un paciente afecto de Klinefelter, sin alteraciones fenotípicas evidentes, que en lugar de tener una disminución del tamaño testicular presentó un aumento de ambos.

La aparición de quistes epidérmicos testiculares sí que se ha relacionado ampliamente con la presencia de testes criptorquídicos $(2 \%$ de los quistes epidermoides).

La orquiectomía ha sido el tratamiento más usado en estos de tumores aunque en algunas ocasiones se ha realizado únicamente enucleación o escisión de la lesión, sobre todo en casos bilaterales o de teste único. En la edad pediátrica el 30\% de tumoraciones testiculares son benignas y no se ha descrito malignización del teratoma (tumoración de difícil diagnóstico diferencial histológico en algunas ocasiones), por lo que Ross et $\mathrm{al}^{1}$ abogan que el tratamiento de elección en los pacientes pediátricos sea la excisión tras exploración inguinal y biopsia por congelación. En la actualidad aún persiste la controversia en el tratamiento, realizando cada cirujano orquiectomía o excisión simple según las características del paciente ${ }^{5}$, pero el tratamiento quirúrgico conservador parece ser la opción más razonable e inocua $^{6}$. En nuestro caso, dado que la lesión era bilateral y en un síndrome de Klinefelter, optamos por esperar a que el paciente cumpla 12 años para valorar orquiectomía, colocación de prótesis y tratamiento androgénico sustitutivo. Dado 


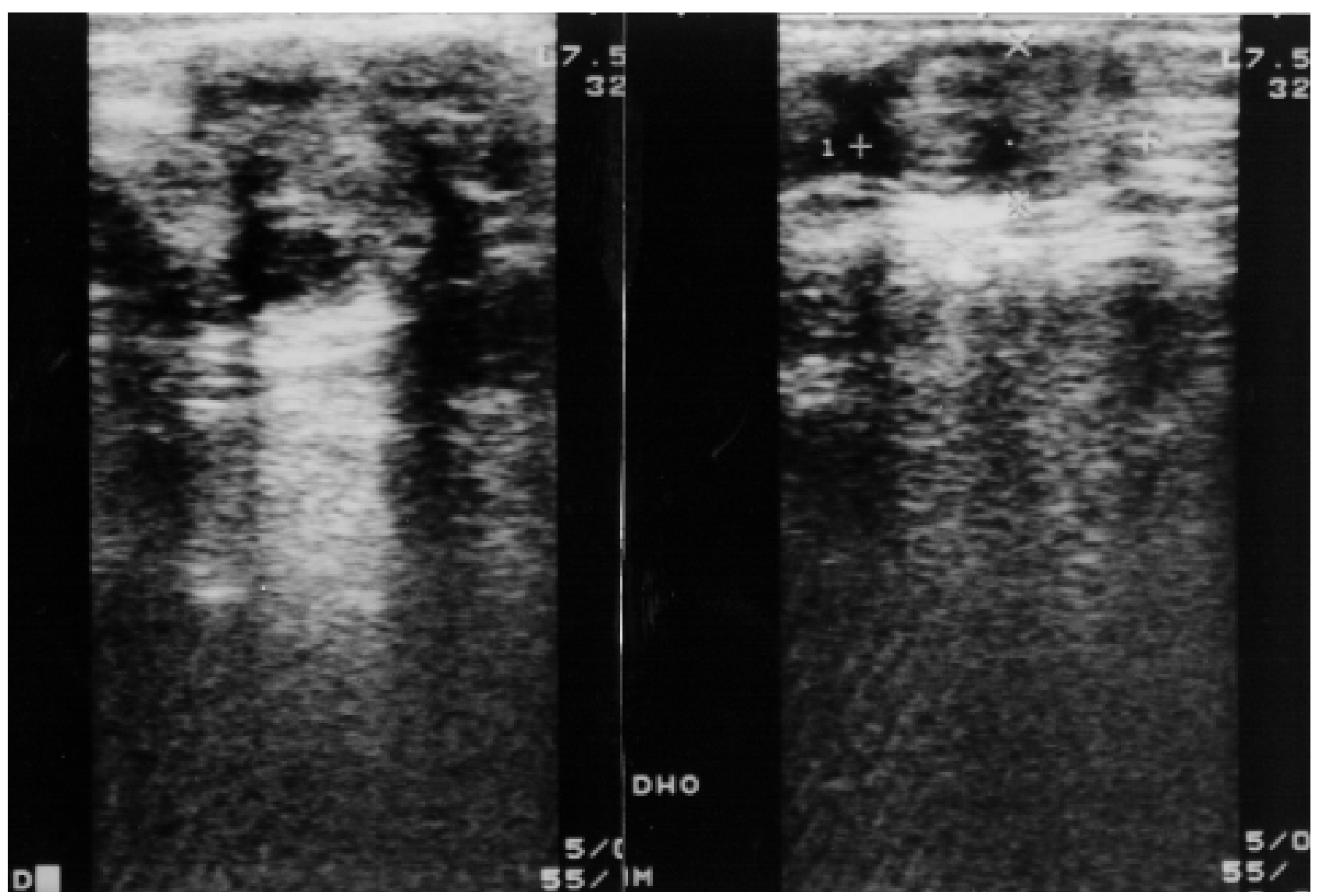

FIGURA 2. A: teste izquierdo; B: teste derecho. Aumento de tamaño del testiculo izquierdo y desestructuración bilateral del parénquima testicular.

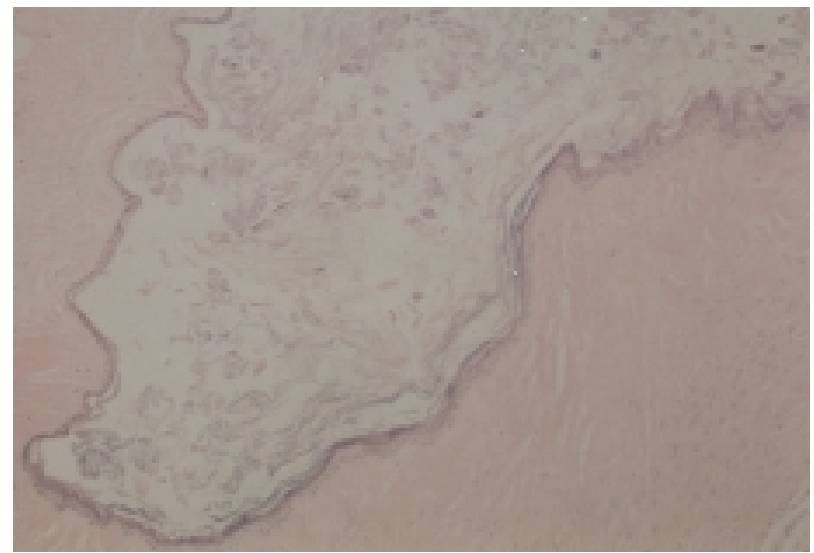

FIGURA 3. Histologia: pared fibrosa delimitando una formación quistica revestida internamente por un epitelio escamoso, estando la cavidad parcialmente rellena por material queratinico. HE 250x.

la benignidad de la tumoración pensamos que el tratamiento debe de ser espectante hasta la pubertad, ya que en caso de orquiectomía el tamaño de la prótesis a implantar será el definitivo.

\section{REFERENCIAS}

1. ROSS JH, KAY R, ELDER J.: Testis sparing surgery for pediatric epidermoid cysts of the testis. $J$ Urol 1993; 149: 353-356.

2. RUSHTON HG, BELMAN AB.: Testis-sparing surgery for benign lesions of the prepubertal testis. Urol Clin North Am 1993; 20: 27-37.

3. BANIEL J, PÉREZ JM, FOSTER RS.: Benign testicular tumor associated with Klinefelter?s syndrome. $J$ Urol 1994; 151: 157-158.

4. SASAGAWA I, NAKADA T, KAZAMA T, SAKAMOTO M, SATOMI S, KATAYAMA T.: Epidermoid cyst of the testis in Klinefelter's syndrome. Urol Int 1987; 42 : 398-400.

5. WALSH C, RUSHTON HG.: Diagnosis and management of teratoma and epidermoid cysts. Urol Clin North Am 2000; 27: 509-518.

6. GARRET JE, CARTWRIGHT PC, SNOW BW, COFFIN CM.: Cystic testicular lesions in the pediatric population. J Urol 2000, 163: 928-936.

Dr. J.M. Alapont Alacreu

Servicio de Urología. Hospital Unviersitario La Fe Avda. Campanar 21

46009 Valencia

(Trabajo recibido el 18 diciembre 2002) 